Journal of Acute Disease

journal homepage: www.jadweb.org

\title{
Analysis of American skills and its educational inspiration for Chinese medicine
}

\author{
Yan-Min Gao, Yan-Qi Hu, Chen-Lei Li, Xiao-Long Qin* \\ School of Clinical Medicine, Shanghai University of Medicine and Health Sciences, Shanghai, 201318, China
}

\begin{tabular}{|c|c|}
\hline ARTICLE INFO & ABSTRACT \\
\hline $\begin{array}{l}\text { Article history: } \\
\text { Received 22 Dec } 2016 \\
\text { Accepted 26 Dec } 2016 \\
\text { Available online 23 Jan } 2017\end{array}$ & $\begin{array}{l}\text { There are various modes of American pre-hospital emergency with different rescue levels } \\
\text { which can meet the needs of out-of-hospital rescue in different environments and grades. } \\
\text { Pre-hospital emergency workers are relatively divided into different levels with different } \\
\text { requirements of skills and practicing certificates. These sources and training of pre-hospital } \\
\text { emergency workers of different levels are independent, totally different from those of medical }\end{array}$ \\
\hline $\begin{array}{l}\text { Keywords: } \\
\text { American workers } \\
\text { Skill } \\
\text { Analysis } \\
\text { Inspiration }\end{array}$ & $\begin{array}{l}\text { emergency on the spot, observing and experiencing the classification and education levels of } \\
\text { American pre-hospital emergency, analyzing the system of American pre-hospital emergency } \\
\text { and their requirements for pre-hospital emergency workers' skills, and comparing with the } \\
\text { actual situation of Chinese medicine education, the study showed the training differences } \\
\text { between American pre-hospital emergency workers and Chinese pre-hospital emergency } \\
\text { workers and their advantages in the hope of enhancing the pertinence of training Chinese pre- } \\
\text { hospital emergency workers and providing a new direction for the development of Chinese pre- } \\
\text { hospital emergency system from another view. }\end{array}$ \\
\hline
\end{tabular}

\section{Introduction}

Pre-hospital emergency is a new important branch of modern medicine, which refers to the emergency rescue that is sent to patients after receiving the alarm information. The main purpose of classification system of emergency rescue is to send the treatment to the person who actually needs it at correct time and correct location by proper ways[1]. It has been over 30 years since the classification system of pre-hospital emergency rescue developed in America, which have relatively resulted in the classified training mechanism for American pre-hospital emergency workers. By my personal experience of observation and participation of the pre-hospital emergency and level training education in America

\footnotetext{
*Corresponding author: Xiao-Long Qin, College of Clinical Medicine, Shangha University of Medicine and Health Sciences, Shanghai, 201318, China.

Tels: +86 15921431380 (YM Gao); +86 13162030721 (XL Qin)

E-mails: gaoym@sumhs.edu.cn (YM Gao); qinxl@sumhs.edu.cn (XL Qin)

Foundation Project: Supported by mechanism research of the establishment of qualification of emergency medical personnel in Shanghai, key project of Shanghai education and scientific research development, and education project of philosophy and social science, 2015 (Grant No. A1507).

The journal implements double-blind peer review practiced by specially invited international editorial board members.
}

in 2014, and a combination with the reference of literature materials, this study analyzed the system of American pre-hospital emergency and requirements of skills for pre-hospital emergency workers of different levels and compared with the Chinese prehospital emergency and training mode for talents to discuss its educational inspiration on Chinese pre-hospital emergency.

\section{Modes of pre-hospital emergency system}

At present, most pre-hospital emergency workers in China are those medical staffs on emergency ambulances with certified qualification[2]. Its operation system belongs to the sanitation system and is independent with fire fighting system and police system, which temporarily cooperate with each other only during the rescue[3]. This has limited the employment channel of prehospital emergency workers and greatly decreased the efficiency of rescue, which makes it hard to meet the needs of pre-hospital emergency rescue for patients. A developed system of pre-hospital emergency is the premise to make sure that the patients can receive effective rescue within the shortest time. 
The system of pre-hospital emergency in America is different from that in China. The call number of it is same with the number of fire fighting and police, which is "911", named emergency medical service (EMS) system. The system includes four kinds of pre-hospital emergency, which are fire emergency, third-party emergency, hospital emergency and private emergency service.

\subsection{Fire emergency}

When the fire department in America provides the service of fire fighting, some trained firemen also be in charged with the prehospital emergency service. Firemen arriving at the scene firstly are mostly trained with courses of emergency medical technicians (EMT) and paramedic training. This mode constitutes one of the main emergency modes of pre-hospital emergency in America at present.

\subsection{Third-party emergency}

Vehicles and workers of this mode belong neither to the fire fighting system nor to the hospital. They are hired by the third party for the service of pre-hospital emergency. There is a large number of this mode in America, which is one of the main emergency modes of pre-hospital emergency as well. However, this mode will be affected by the administrative management and municipal budget.

\subsection{Hospital emergency}

Parts of the emergency resources in hospitals are utilized to conduct pre-hospital emergency and transfer critical patients. Some specialized hospitals have their own professional emergency service, such as children's hospital in Milwaukee which has professional pre-hospital emergency ambulance for children and relative workers. Hospital emergency focuses on constant nursing. Uninterrupted electrocardiograph monitoring and mechanical ventilation can be carried out during the process of transfer. Hospital emergency sometimes utilizes faster means of transportation such as helicopters, but that is limited by operating costs.

\subsection{Private emergency}

When companies with private nature provide pre-hospital emergency service, most of their employees are skilled talents graduating from community colleges and majoring in pre-hospital emergency. Ambulances of this mode are not included in the 911 calling system. It provides private rescue and transfer which enables the patients to choose hospitals by themselves, but it costs a lot.

Therefore, at the same time when Chinese pre-hospital emergency attaches importance to the training of emergency treatment and emergency workers, the social emergency power out of hospital system should be fully utilized as well[4], such as the fireman or policeman and school teachers who arrive at the site of accident firstly. The management of Chinese prehospital emergency can take the American modes as reference and adopt classification system, i.e. 120 emergency doctors onboard, emergency assistants and social emergency persons, which are all in the hope of gathering together the social strength to improve the pre-hospital emergency system in China.

\section{Modes of pre-hospital emergency levels and personnel training levels in America}

At present, the pre-hospital emergency system in China has put the large and medium-sized cities at the core of the pre-hospital emergency network. But pre-hospital emergency systems of most cities still rest on simple information collection and mechanical assignment, and does not utilize the grading assignment for prehospital emergency. The assignment of ambulances basically neglect the classification and severity of diseases, which usually results in the late rescue for actual severe patients[2].

However, there are various modes of American pre-hospital emergency with different rescue levels, which can meet the needs of out-of-hospital rescue in different environments and grades. The American pre-hospital emergency has experienced different stages of development[5]. After summarizing the historical experience, they separated the pre-hospital emergency and hospital emergency and set four levels for the pre-hospital emergency system. The sources of the workers with basic rescue skills are rich and they account for a number of people such as emergency medical responders (EMR) and emergency medical technicians-basic (EMT-B). These pre-hospital emergency workers include not only those professional ones, but also firemen, policemen, rescuers, school teachers and so on who have taken professional emergency training. It is characterized by the large number and wide range of distribution, which can quickly react with the emergency scene and save time for the high-level medical emergency.

The pre-hospital emergency workers are relatively graded into different levels, which are required different kinds of skills and practicing certificates according to the different levels. The sources and training of these pre-hospital emergency workers from different levels are independent, totally different from those medical care staffs in hospital[6,7]. The emergency levels of American prehospital emergency system from low to high are EMR, EMT, 
advanced emergency medical technician and paramedic.

Specific training schemes and training time schedules are given to pre-hospital emergency workers of different levels, and the mergency operations of pre-hospital emergency workers of different levels are different as well (Figure 1)[8].

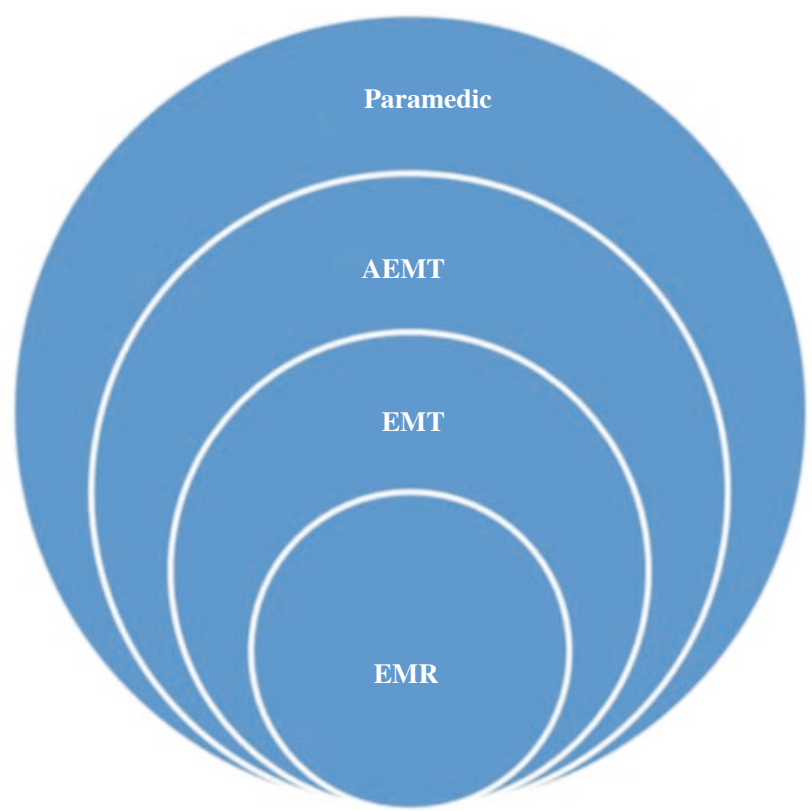

Figure 1. Levels of skill requirements for American pre-hospital emergency workers.

AEMT: Advanced emergency medical technician.

\section{1. $E M R$}

The most basic emergency workers include professional prehospital emergency workers, policemen, rescuers, school teachers and so on. These workers are required to take basic professional emergency training for 48-60 h. The skills required for the EMR level include the environment assessment, assessment of vital signs, cardio-pulmonary resuscitation, use of automated external defibrillator, fracture fixation, binding up and patients transfer, etc., which are all non-invasive operations. However, EMR can only use automatic injection of adrenaline and oral glucose. That is greatly limited.

\section{2. $E M T-B$}

Many young Americans become firemen in fire department after graduating from high school and receiving professional training, and some of them will choose to attend community colleges to receive 400-hour EMT training courses due to the sense of responsibility and quite favorable salary. EMT is the first active team for 911 calls, whose skill requirements include hemostasis, face mask, mouth (nose) and throat airways, as well as the use of splint including whole vertebra fixation based on the requirements of EMR.

\subsection{Emergency medical technicians-basic (EMT-B) or emergency medical technicians-advanced (EMT-A)}

EMT-B or EMT-A is the pre-hospital emergency level between EMT and EMT-paramedic. All states in America give different standards and courses on this level with a training time of 500-600 h. Some courses on this level are taught by the training institution of government pre-hospital emergency, i.e. the EMS county. Skill requirements including vein patent and the use of a few medicines are added for EMT-A on the basic of the requirments of EMT-B.

\subsection{EMT-paramedic}

Generally. the fire department will assign EMT who have firstly passed the EMT-I course to learn EMT-paramedic courses in EMS county. Those who pass the course will become the paramedic staffs with high-level skills. And all the training fees are charged from the government. Paramedic courses have two types. One is opened from October to September, with two-day classes per week and $8 \mathrm{~h}$ per day. The other one is opened from January to April each year, with classes from Monday to Friday and $8 \mathrm{~h}$ per day. The total study time of paramedic courses is $650 \mathrm{~h}$, and along with the courses of EMT and EMT-I, it could be $1400 \mathrm{~h}$ in total. The greatest feature of paramedic courses is that the time of practice, hospital and onboard practice is much longer. Paramedic workers can carry out some invasive operation besides the basic life support (BLS) operation, including intravenous administration and trachea cannula, and even they are allowed to use over 30 oral and venous medicines[9].

In America, basic medical emergency service can be conducted by firemen, policemen and so on. Meanwhile, the pre-hospital emergency workers can be from different social groups. As long as they finish the relative training level and pass the examination, they can all provide pre-hospital emergency service. This training mode enhances the efficiency of talent training for pre-hospital emergency, and decreases the training cost as well. The training mode of Chinese pre-hospital emergency can take the modes of level training of American pre-hospital emergency as reference to establish pre-hospital emergency as a profession. Emergency workers of different levels should relatively have the corresponding lowest educational level and different ways of training. The results of this study indicated that the lowest educational background of social emergency workers can be unlimited. Emergency assistants should have high vocational diploma and the emergency physicians should be full-time undergraduate. In terms of the ways of training, social emergency workers can be obtained by short-term training. Emergency assistants should receive continuing education, while emergency physicians should have full-time clinical medicine 
education.

\section{Examination for pre-hospital emergency workers}

There are two types of practicing certificates for American pre-hospital emergency workers, which are national certificate and state certificate. The distinction between them is their practicing scopes, which means that the state practicing certificate only allows the workers to work on pre-hospital emergency within the state, while national practicing certificate allows them to work on pre-hospital emergency in any states in America. Both practicing certificates are examined by relevant government departments, but the contents of the examination of national practicing certificate will be much more and the scope involving is wider. However, there is no specific qualification test for pre-hospital emergency physicians and examination for practice in China at present. Pre-hospital emergency workers are mainly medical personnel who experience 5-year full-time undergraduate education and pass the national assessment of medical practitioners/nurse, and the contents of the examination are same as those of specialist physicians, which shows some certain differences with the post skill of pre-hospital emergency. For example, the examination of practicing physicians requires a total understanding of the pathogenesis and main points of diagnose of various clinical diseases, while the pre-hospital emergency system does not require such comprehensive and profound knowledge. The pre-hospital emergency system requires the workers to know emergency skills such as the airway management and electric defibrillation, which are not included in the examination of practicing physicians. Therefore, we should use the assessment mode of America for reference in the hope of developing an assessment mode that is suitable for the position requirements of pre-hospital emergency in China.

\section{Conclusion}

The development of pre-hospital emergency and personnel training in China cannot be achieved overnight. It needs to combine with the China's national condition, take the level system and training mode of pre-hospital emergency in America as reference to realize the development step by step.

\section{Conflict of interest statement}

The authors report no conflict of interest.

\section{References}

[1] Clawson JJ. Running "hot" and the case of Sharron Rose. JEMS $1991 ; 16(7): 11-3$

[2] Lu F, Li MH, Wu DG, Zhang Y. [The present situation of prehospital emergency category ambulance service system in the world and the prospects of its application in China]. Chin Health Res 2013; 16(1): 74-6. Chinese.

[3] Peng BB, Zheng JC, Liu HF. [Enlightenment of worldwide development of training and practicing system of emergency medical technician on China]. Chin J Disaster Med 2016; 4(1): 2-5. Chinese.

[4] Han Y, Chen XF, Lin WD. [The expansion and extension of the fire field medical aid to the current pre-hospital first aid in our country]. Chin J Diagnostics (Electronic Edition) 2016; 4(1): 33-5. Chinese.

[5] Wikipedia. Emergency medical technician. Tampa: Wikipedia. 2006. [Online] Available from: https://en.wikipedia.org/wiki/ Emergency_medical_technician [Accessed on 25th November, 2016]

[6] Cai ZB. [Current situation of pre-hospital emergency domestic and abroad]. Chin J Emerg Med 2010; 19(7): 775-7. Chinese.

[7] U.S. Department of Transportation, National Highway Traffic Safety Administration. National emergency medical services education standards. Florida: Progressive Management; 2012.

[8] National Highway Traffic Safety Administration. The National Emergency Medical Services Scope of Practice Model Table of Contents. 2007, DOT HS810657.

[9] National Highway Traffic Safety Administration. National EMS scope of practice model. Washington, D.C.: National Highway Traffic Safety Administration. [Online] Available from: http:// www. ems.gov/education/EMSScope.pdf [Accessed on 12th December, 2016] 